\title{
Plasma edge transport phenomena caused by particle drifts and sources in TEXTOR
}

\author{
M. Lehnen, M. Brix, U. Samm, B. Schweer, B. Unterberg and \\ the TEXTOR-team \\ Institut für Plasmaphysik, Forschungszentrum Jülich GmbH, EURATOM Association, \\ Trilateral Euregio Cluster, D-52425 Jülich, Germany
}

Received 16 May 2002, accepted for publication 16 January 2003

Published 13 February 2003

Online at stacks.iop.org/NF/43/168

\begin{abstract}
The parallel and radial transport properties of the plasma edge of TEXTOR are studied using radial electron density and temperature profiles as well as ion temperatures and poloidal velocities in the scrape-off layer (SOL). These quantities are measured by thermal helium beams at the low (LFS) and high field side (HFS) by emission and beam driven charge exchange recombination spectroscopy. We investigate the influence of the safety factor and of a magnetic field reversal on these edge parameters. Especially the field reversal leads to clear effects: a decrease of the density at the LFS, a significant change in the poloidal density distribution, which is identified by comparing LFS and HFS densities, and an increase in the density e-folding length at both poloidal positions. The poloidal ion rotation changes sign in reversed field configuration and thus gives a hint on the role of poloidal drifts in the SOL. For further analysis, we present a simple fluid model including poloidal drift velocities and local ionization sources. With this model we can show that the poloidal $E \times B$ drift clearly influences the poloidal density distribution. However, although the model results show the same tendencies as the experimental findings, the impact of the field reversal on the density asymmetry is not that pronounced as in the experiment. The dependence of the density e-folding length on the poloidal and radial drifts as well as on the source distribution is discussed within the frame of analytical estimates.
\end{abstract}

PACS numbers: 52.25.Fi, 52.55.Fa, 52.65.Kj

\section{Introduction}

The transport in the scrape-off layer (SOL) of a tokamak is supposed to be significantly affected by drift motions and particle sources. Investigations of the poloidal and radial structure in various tokamaks show that the SOL transport is influenced by strong mechanisms. Examples for this are particle and heat flux in-out asymmetries in a divertor [1-3] or poloidal asymmetries of radial density profiles in the limiter SOL $[4,5]$. The observation of large parallel Mach numbers in the SOL far away from the divertor plates [6] as well as the measurements of the poloidal plasma rotation [5] suggest that drifts might play an important role in the SOL. The control of poloidal plasma rotations for example is an important element for the improvement of particle exhaust in limiter biasing experiments [7]. However, a complete picture to explain all experimental observations is still missing. Especially the high parallel flows in the divertor SOL cannot be satisfactorily explained by the existing edge drift models $[6,8]$. The topic of this paper is the study of drifts and particle sources with respect to their influence on the limiter SOL. The densities and temperatures in the SOL and their radial evolution (especially the SOL width) as well as the poloidal structure of the SOL is investigated. We concentrate especially on the effects of the poloidal $E \times B$ drift and the influence of local particle sources. The experimental findings are analysed using a simplified fluid model, which includes these two mechanisms.

The important drift motions in the tokamak edge are the $E \times B$ drift and the diamagnetic $\nabla p \times B$ drift. The radial $E \times B$ drift is strongest in the vicinity of the limiter, where one expects a large poloidal electric field. It has opposite sign on each side of the limiter (cf figure 1). The poloidal $E \times B$ drift is larger due to the steeper gradients of the plasma potential in radial direction. It is expected to range up to about $3000 \mathrm{~m} \mathrm{~s}^{-1}$ in the TEXTOR SOL. In normal magnetic field configuration it is directed from the bottom part of the toroidal limiter along the SOL to the top of the limiter as shown in figure 1. The diamagnetic drift splits into a divergence-free part, which leads to radial and poloidal drift velocities, and into a part due to the magnetic field gradient and curvature resulting in vertical drift motions. The divergence-free part of the diamagnetic drift does not contribute to particle transport. The ion drift velocities depend on the radial pressure gradient and reach up to $6000 \mathrm{~m} \mathrm{~s}^{-1}$ in poloidal direction, the radial velocity is of the same order as for the $E \times B$ drift. The second part of the diamagnetic drift (arising from $\nabla B$ ) results 
in vertical drift motions of the order of about $50 \mathrm{~m} \mathrm{~s}^{-1}$ in the TEXTOR SOL. Its contribution to the poloidal drift can be neglected. However, this drift might give rise to PfirschSchlüter currents. Their influence on SOL asymmetries are for example discussed in [9]. The direction of all these drifts depends on the orientation of the magnetic field, so that their influence on the edge plasma can be investigated by a magnetic field reversal. The drift velocity can be actively controlled by changing the e-folding lengths of the SOL temperature [5] or by changing the SOL pressure gradient. We will focus in this paper on the dependence of the edge temperature and density and their e-folding length on the safety factor and on magnetic field reversal.

In the first part of this paper, we will report on the experimental findings (section 2). We have measured profiles of electron density and temperature as well as the ion temperature and the poloidal rotation in the plasma edge by means of thermal helium beams at the low (LFS) and high field side (HFS). A short description of the experimental setup will be given. We varied the safety factor by changing either the toroidal magnetic field or the plasma current. The field reversal was performed by changing the sign of both plasma current and toroidal field, in order to preserve the magnetic geometry. The edge parameters at the last closed flux surface (LCFS) are given as a function of the safety factor.

The second part of the paper is dedicated to the introduction of a simplified SOL model (section 3). We included local particle sources and poloidal drifts in this model, but tried to keep it still as simple as possible. This means that the fluid equations are solved only in poloidal direction and that the neutral model is based on simple analytical expressions. With the help of this model the experimental findings are analysed especially with regard to drift effects (section 4). Various mechanisms, which can influence the SOL width, are discussed with the help of analytical estimates.

\section{Experiment}

\subsection{Setup}

The tokamak TEXTOR is a medium size device with a pumped toroidal belt limiter at a poloidal angle of $\theta=-45^{\circ}$ (see figure 1). The major radius of the machine is $R_{0}=1.75 \mathrm{~m}$, the minor radius is $a=0.46 \mathrm{~m}$. We will present discharges with strong heating by neutral beam injection in co-direction to the plasma current with a power of $1.35 \mathrm{MW}$. The variation of the safety factor has been achieved by varying the central toroidal field $B_{\phi}$ between 1.8 and $2.6 \mathrm{~T}$ or by varying the plasma current $I_{\mathrm{P}}$ between 280 and $450 \mathrm{kA}$. All discharges were made at a line averaged density of $\bar{n}_{\mathrm{e}}=3.5 \times 10^{19} \mathrm{~m}^{-3}$. During the experiments with field reversal both $B_{\phi}$ and $I_{\mathrm{P}}$, have been reversed in order to maintain the magnetic geometry.

\subsection{Diagnostic}

The edge parameters discussed in this paper were measured by the helium beam diagnostic [10]. Neutral helium is injected at two poloidal positions - at the LFS and at the HFS - for a local measurement of the electron temperature $T_{\mathrm{e}}$ and density $n_{\mathrm{e}}$. Three helium transitions are observed at $\lambda_{1}=667.8$,

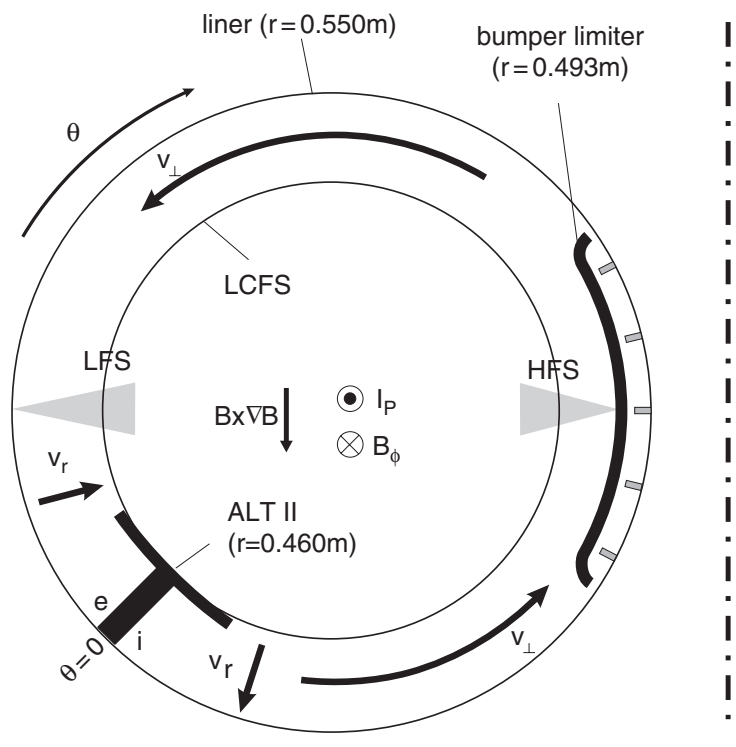

Figure 1. Poloidal cross-section of TEXTOR indicating the two locations of the helium beam. The ion drift motions in the SOL are indicated for the normal magnetic field configuration.

$\lambda_{2}=706.5$ and $\lambda_{3}=728.1 \mathrm{~nm}$. The line intensity ratios $\lambda_{1} / \lambda_{3}$ and $\lambda_{3} / \lambda_{2}$ are compared to a collisional-radiative model to give $n_{\mathrm{e}}$ and $T_{\mathrm{e}}$. The achievable radial resolution of less than $1.5 \mathrm{~mm}$ is well below the width of the SOL so that the radial structure of the plasma edge can be analysed.

Measurements of the poloidal plasma rotation and the ion temperature were done at the LFS exploiting the thermal helium beam for charge exchange recombination spectroscopy (CXRS). The measurement of the spectral position and of the shape of the $\mathrm{C}$ vi line at $\lambda=529 \mathrm{~nm}$ with a high resolution $\left(\lambda / \Delta \lambda=1.5 \times 10^{5}\right)$ spectrometer gives us values for the poloidal velocity of $\mathrm{C}^{6+}$ from the Doppler shift and the ion temperature from the Doppler broadening of that line [11]. Possible deviations between the velocity of $\mathrm{C}^{6+}$ and the hydrogen background are discussed in section 4.4. The line shape has been corrected with respect to the Zeeman splitting and the fine structure [12]. The spectral calibration was done in [11] relative to a molecular line, here we use an observation of the helium beam with two opposite lines of sight to find the unshifted wavelength of $\mathrm{C}$ VI for the velocity measurement. Both quantities, $v_{\theta}$ and $T_{\mathrm{i}}$, are measured inside the SOL at $r=0.466 \mathrm{~m}$ averaged over $\Delta r=0.005 \mathrm{~m}$.

\subsection{Density and temperature}

The electron temperature and density at the LCFS as well as the ion temperature $6 \mathrm{~mm}$ inside the SOL are plotted in figure 2 for the current and the field scan. The edge parameters are given for discharges with normal and reversed magnetic field orientation. Beside the experimental values, the results from the SOL model discussed in the second part of the paper are included in these plots as lines. The model is introduced in section 3 and the model results will be discussed in section 4 .

The ion and electron temperature in both scans do not show a clear dependence on $q$. In contrast, the density shows a significant different behaviour between the current and the field scan. The decrease of the plasma current leads to an 

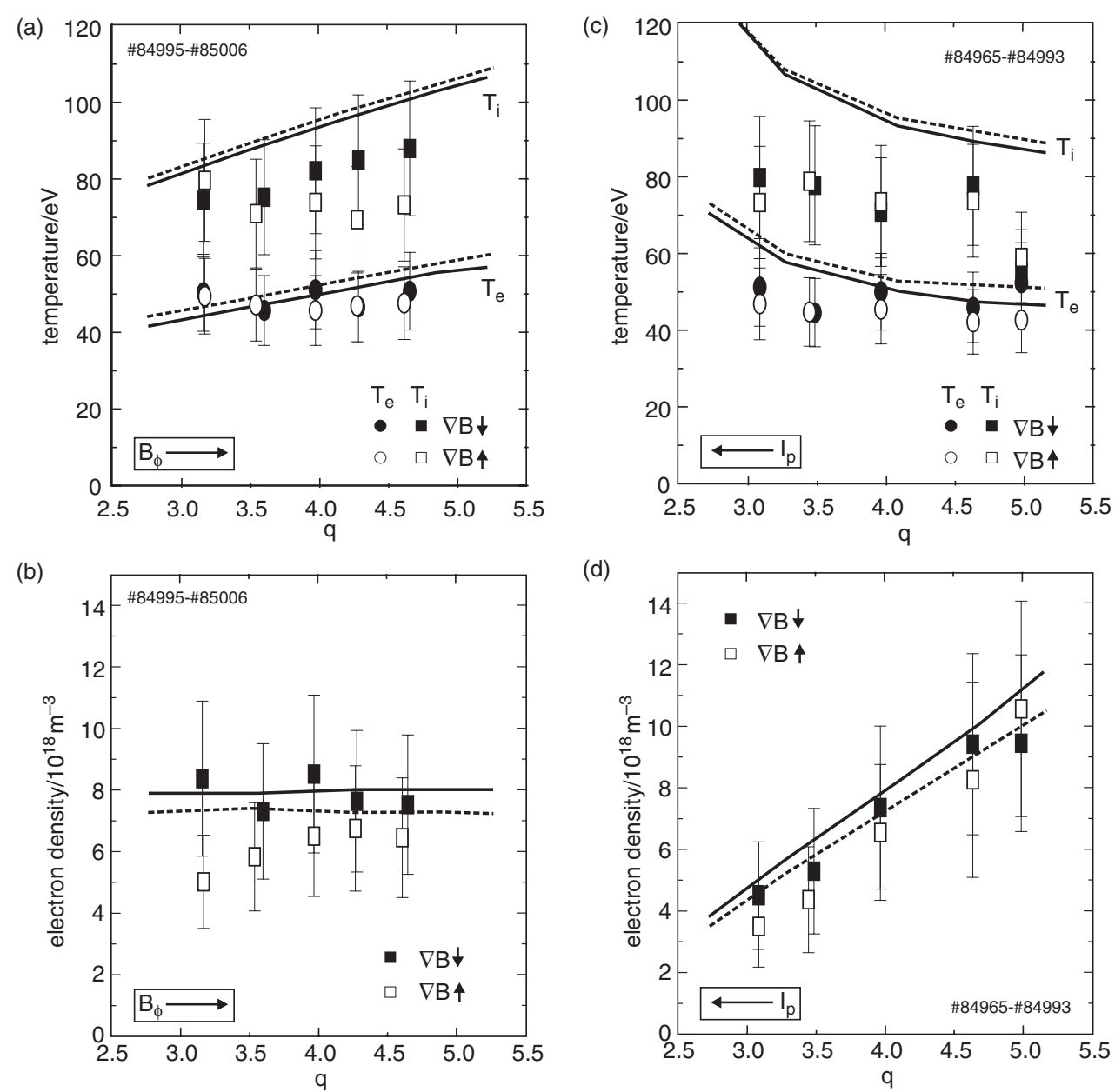

Figure 2. Edge parameters electron temperature and density on the LFS at the LCFS ( $\left.R-R_{0}=460 \mathrm{~mm}\right)$ and ion temperature on the LFS in the SOL $\left(R-R_{0}=466 \mathrm{~mm}\right):(a)$ and $(b)$ variation of $B_{\phi} ;(c)$ and $(d)$ variation of $I_{\mathrm{P}}$. The lines are the model results (see section 3$)$. Dashed lines refer to the reversed field configuration.

increase of the edge density whereas the variation of $B_{\phi}$ does not change the density. The strong dependence of the edge density on the plasma current comes from the changes in the confined plasma, i.e. inside the LCFS. The plasma density is controlled by the line averaged density measured by the central interferometer channel. A more box-shaped core density profile with steeper gradients at the edge-as it is the case for high plasma currents-leads to a decrease in the edge density if the line average is kept constant. This effect is also seen on the ion flux to the limiter deduced from $D_{\alpha}$ light, which doubles going from high to low plasma current.

The magnetic field reversal does have an influence on the edge density. The density at the LFS is smaller in the case of a reversed field. Although this is a small effect of about $10 \%$ this change in density happens systematically and can be seen for both $I_{\mathrm{P}}$ and $B_{\phi}$ scan. The temperatures show no clear dependence on the magnetic field direction.

\subsection{Radial structure}

We discuss the radial structure in the SOL in terms of the e-folding length of the electron density and temperature. These quantities are given in figure 3 . The density e-folding length $\lambda_{n}$ is shown for both the LFS and the HFS. The measurement of the electron temperature at the HFS is subject to large errors which are due to the special diagnostic arrangement. Thus the e-folding length $\lambda_{\mathrm{e}}$ can only be shown for the LFS.

The density e-folding length increases for both scans, $I_{\mathrm{P}}$ and $B_{\phi}$, with the safety factor $q$. This correlates with the increasing connection length. The ratio of $\lambda_{n}$ between HFS and LFS is around 1.4. This is clearly expected from the different radial separation of the flux surfaces at both poloidal positions due to the Shafranov shift. The e-folding length of the temperature shows no clear dependence on $q$.

The influence of the field reversal on $\lambda_{n}$ is small, butas in the case of the edge density - has a clear tendency to increase $\lambda_{n}$ by $10-20 \%$. Such a tendency is not seen for $\lambda_{\mathrm{e}}$.

\subsection{Plasma rotation}

We measured the poloidal velocity $v_{\theta}$ of $\mathrm{C}^{6+}$ by CXRS with the thermal helium beam at the LFS. This poloidal velocity consists of the diamagnetic rotation and the $E \times B$ rotation of the carbon ions. Moreover, the poloidal projection of the parallel velocity adds to $v_{\theta}$. Figure 4 shows the poloidal velocity for both scans and field directions. We find a negative $v_{\theta}$ for normal field configuration, which corresponds to a 

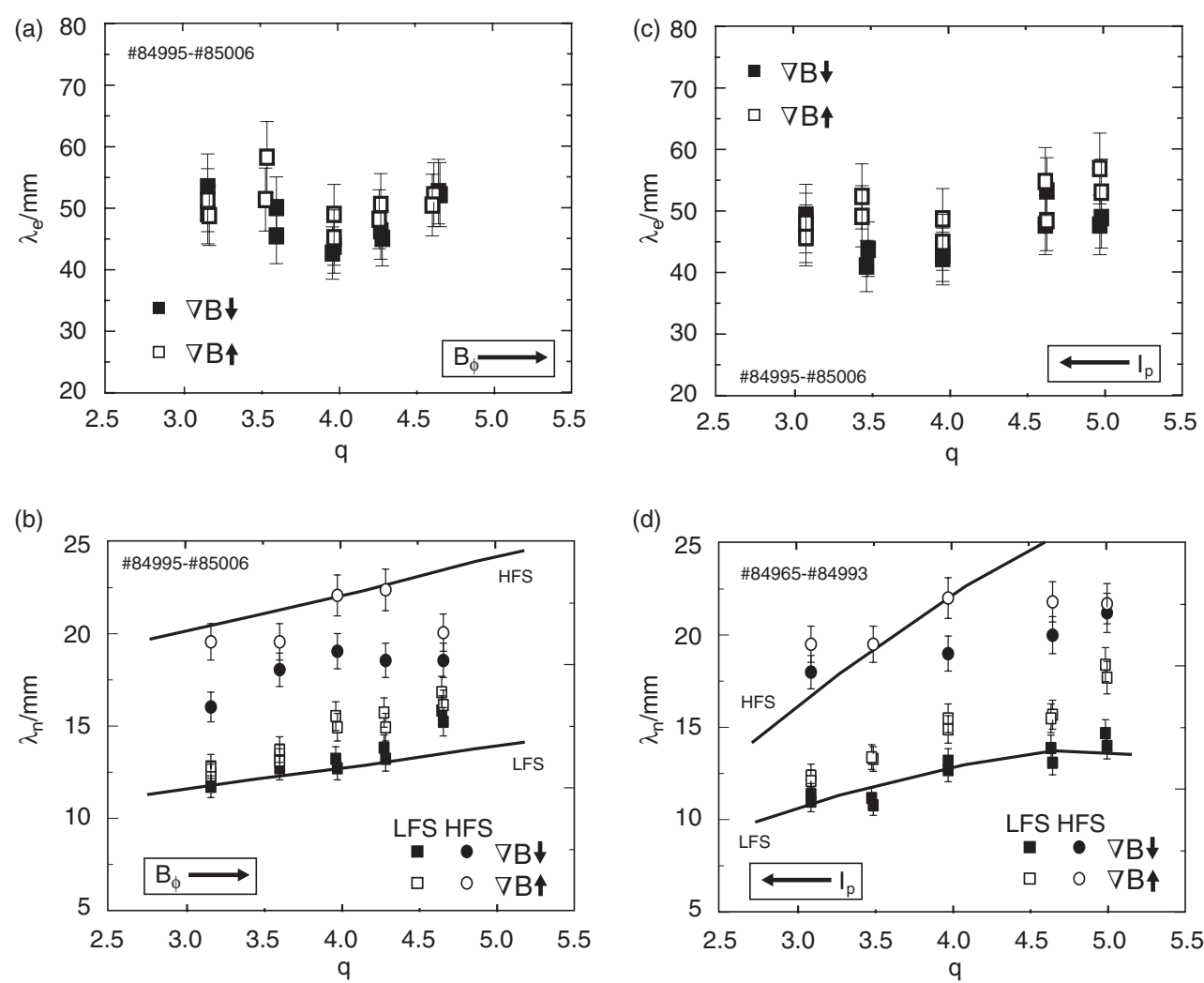

Figure 3. E-folding length of the electron temperature $\lambda_{\mathrm{e}}$ at the LFS and density $\lambda_{n}$ at the LFS and HFS in the SOL as a function of $q$ : (a) and $(b)$ variation of $B_{\phi} ;(c)$ and $(d)$ variation of $I_{\mathrm{P}}$. The lines are the model results (see section 3 ). The value of the modelled $\lambda_{n}$ does not change with field reversal.

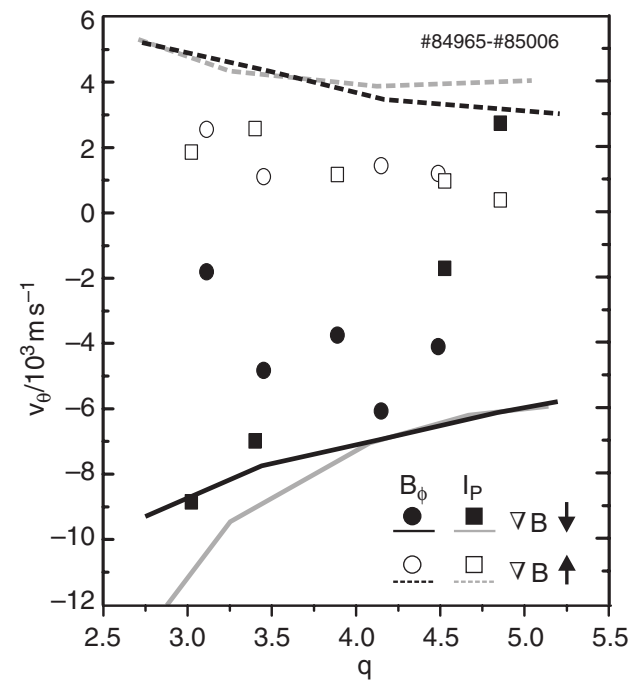

Figure 4. Poloidal velocity of $\mathrm{C}^{6+}$ (TEXTOR) and $\mathrm{D}^{+}$(SOL model) The dashed lines refer to reversed field configuration, the solid lines to the normal configuration. Black lines refer to the $B_{\phi}$ scan, grey lines to the $I_{\mathrm{P}}$ scan.

counter-clockwise plasma rotation in the view of figure 1, i.e. from the ion side of the limiter to the electron side. Field reversal leads to a change of sign in the poloidal velocity. The absolute values of $v_{\theta}$ are larger for the normal configuration compared to the reversed case.

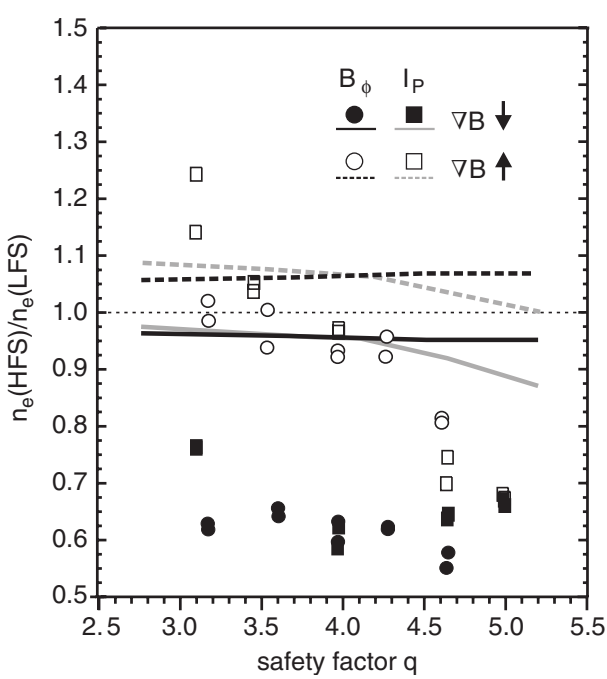

Figure 5. Density ratio at the LCFS between HFS and LFS versus the safety factor. The dashed lines (reversed field configuration) and the solid lines (normal configuration) are the model predictions. Black lines refer to the $B_{\phi}$ scan, grey lines to the $I_{\mathrm{P}}$ scan.

\subsection{Poloidal asymmetries}

Beside the fact that we clearly see the influence of the Shafranov shift on the density e-folding lengths at the two poloidal positions LFS and HFS, also the density itself shows a strong poloidal variation. The density ratio $n_{\mathrm{e}}(\mathrm{HFS}) / n_{\mathrm{e}}(\mathrm{LFS})$ at the LCFS is plotted in figure 5. In normal field configuration, 
the density at the LFS is higher compared to the HFS by about a factor of 1.4-1.6. The density ratio changes strongly with reversed field and tends to decrease with increasing safety factor. We do not see any significant change in the density ratio due to the density increase during the $I_{\mathrm{P}}$ scan.

From the experimental point of view, the determination of the LCFS at the HFS is not straightforward. The plasma position is controlled by comparing the density at an inner and an outer position of the plasma column. Strong changes in beta poloidal-as it happens especially during a scan of the plasma current-change the Shafranov shift and thus lead to a shift of the position of the LCFS. At the LFS, this position is more fixed due to the toroidal limiter. We assume that the plasma parameters do not change on the flux surface $10 \mathrm{~mm}$ inside the LCFS. The density measured at the LFS on this flux surface can then be used to find the same surface at the HFS. This flux surface is used as a reference at the HFS and the position of the LCFS is given relative to it.

\section{SOL model}

We present in this section a simplified SOL model, which is based on the fluid equations by Braginskii [13]. The equations are solved in the poloidal direction. However, we include the radial influxes of particles and energy as source terms in the equations. Poloidal drift motions due to $E \times B$ drifts are included. Currents are neglected and also viscosity is not taken into account. The neutral model is based on simple analytical expressions.

\subsection{Basic equations}

We take the fluid equation as they can be found in [14] (equations (3.6)-(3.10)). The equations for particle conservation, momentum and energy look as follows:

$$
\begin{gathered}
\frac{1}{H} \frac{\partial}{\partial \theta}\left(\frac{H}{H_{\theta}} n v_{\theta}\right)+\frac{1}{H} \frac{\partial}{\partial r}\left(\frac{H}{H_{r}} \Gamma_{r}\right)=S_{\mathrm{p}}, \\
\frac{1}{H} \frac{\partial}{\partial \theta}\left(\frac{H}{H_{\theta}} m n v_{\theta} v_{\|}\right)=-\frac{B_{\theta}}{B} \frac{1}{H_{\theta}} \frac{\partial p}{\partial \theta}+S_{\mathrm{m}}, \\
\frac{1}{H} \frac{\partial}{\partial \theta}\left(\frac{H}{H_{\theta}} \frac{5}{2} n v_{\theta} T_{\mathrm{e}}-\frac{H}{H_{\theta}^{2}} \frac{B_{\theta}^{2}}{B^{2}} \kappa_{\mathrm{e} \|} \frac{\partial T_{\mathrm{e}}}{\partial \theta}\right)+\frac{1}{H} \frac{\partial}{\partial r}\left(\frac{H}{H_{r}} Q_{r \mathrm{e}}\right) \\
=-Q_{\mathrm{ei}}+S_{E \mathrm{e}}+\frac{v_{\theta}}{H_{\theta}} \frac{\partial p_{\mathrm{e}}}{\partial \theta}, \\
\frac{1}{H} \frac{\partial}{\partial \theta}\left(\frac{H}{H_{\theta}}\left(\frac{5}{2} T_{\mathrm{e}}+\frac{1}{2} m v_{\|}^{2}\right) n v_{\theta}-\frac{H}{H_{\theta}^{2}} \frac{B_{\theta}^{2}}{B^{2}} \kappa_{\mathrm{i} \|} \frac{\partial T_{\mathrm{e}}}{\partial \theta}\right) \\
+\frac{1}{H} \frac{\partial}{\partial r}\left(\frac{H}{H_{r}} Q_{r \mathrm{i}}\right)=Q_{\mathrm{ei}}+S_{E \mathrm{i}}-\frac{v_{\theta}}{H_{\theta}} \frac{\partial p_{\mathrm{e}}}{\partial \theta} .
\end{gathered}
$$

The radial term in the momentum equation is omitted, viscosity is not taken into account. The radial particle flux $\Gamma_{r}$ is given by

$$
\Gamma_{r}=\frac{D}{H_{r}} \frac{n}{\lambda_{n}}
$$

where we assume a diffusive transport with diffusion coefficient $D$ and an e-folding length of the density $\lambda_{n}$. From this we get for the radial derivative of $\Gamma_{r}$ (second term on the left-hand side of equation (1))

$$
\frac{1}{H} \frac{\partial}{\partial r}\left(\frac{H}{H_{r}} \Gamma_{r}\right)=\frac{D}{H_{r}^{2}} \frac{n}{\lambda_{n}^{2}} .
$$

The value $D / \lambda_{n}^{2}$ follows from the boundary conditions for the velocity at both sides of the limiter. It is thus determined by the poloidal connection length $L_{\theta}=2 \pi a$.

The radial heat flux $Q_{r}$ is given by

$$
Q_{r}=\frac{1}{H} \frac{\partial}{\partial r}\left(\frac{H}{H_{r}} \frac{5}{2} \Gamma_{r} T-\frac{H}{H_{r}^{2}} \kappa_{r} \frac{\partial T}{\partial r}\right) .
$$

We prescribe a constant energy influx for electrons $Q_{\mathrm{e}}=$ $H_{r} Q_{r \mathrm{e}}$ and ions $Q_{\mathrm{i}}=H_{r} Q_{r \mathrm{i}}$, which is determined by the heating power which enters the SOL: $P_{\mathrm{SOL}}$. Replacing the radial derivatives with the e-folding lengths for the electron temperature $\lambda_{\mathrm{e}}$ and the ion temperature $\lambda_{\mathrm{i}}$ makes $Q_{r}$ proportional to the plasma pressure. Thus, we can write the radial derivative (second term on the left-hand side of equations (3) and (4)) like

$$
\frac{1}{H} \frac{\partial}{\partial r}\left(\frac{H}{H_{r}} Q_{r \mathrm{e}, \mathrm{i}}\right)=\left(\frac{1}{\lambda_{n}}+\frac{1}{\lambda_{\mathrm{e}, \mathrm{i}}}\right) \frac{1}{H_{r}^{2}} Q_{\mathrm{e}, \mathrm{i}} .
$$

The source terms on the right-hand side of equations (3) and (4) are the energy exchange between ions and electrons $Q_{\text {ei }}$ and the energy source or sink due to neutral interaction $S_{E \mathrm{e}}$ and $S_{E \mathrm{i}}$. The former source is defined as

$$
Q_{\mathrm{ei}}=3 \frac{m_{\mathrm{e}}}{m_{\mathrm{i}}} n\left(T_{\mathrm{i}}-T_{\mathrm{e}}\right) v_{\mathrm{e}},
$$

where $v_{\mathrm{e}}$ is the electron collision frequency. The latter sources are due to ionization and charge exchange:

$$
\begin{gathered}
S_{E \mathrm{e}}=S_{\mathrm{P}} E_{\mathrm{ion}}, \\
S_{E \mathrm{i}}=S_{\mathrm{P}} T_{0}+n_{0} n\left\langle\sigma_{\mathrm{cx}} v\right\rangle k\left(T_{0 \mathrm{~s}}-T_{0 \mathrm{f}}\right),
\end{gathered}
$$

with $E_{\text {ion }}=-25 \mathrm{eV}$.

Within this model, we take also the toroidal geometry into account. We choose the metric coefficients as

$$
\begin{gathered}
H_{\phi}=R, \quad H_{\theta}=a, \quad H_{r}=1-2 \frac{\delta_{0}}{a} \cos \left(\theta-\frac{\pi}{4}\right) \\
\text { and } \quad H=H_{\phi} H_{\theta} H_{r},
\end{gathered}
$$

with major radius $R=R_{0}+a \cos (\theta)$. The circular plasma shape of TEXTOR gives an easy opportunity to include the Shafranov shift inside the metric coefficients. We take a parabolic shape of the radial profile of the Shafranov shift $\delta(r)$

$$
\delta(r)=\delta_{0}\left(1-\frac{r^{2}}{a^{2}}\right)
$$

and get $H_{r}$ from

$$
H_{r}=1-\frac{\partial \delta}{\partial r}(a) \cos \left(\theta-\frac{\pi}{4}\right) .
$$

The Shafranov shift itself is extracted from a scaling found from equilibrium calculations: $\delta_{0}=\left(23+60 \beta_{\mathrm{p}}\right) \mathrm{mm}$ [15]. The beta poloidal $\beta_{\mathrm{p}}$ is about 0.65 for the magnetic field scan, but changes strongly during the current scan $\left(\beta_{\mathrm{p}} \approx 0.45 \ldots 0.8\right)$. 
The poloidal drift velocity $v_{\perp}$ enters the equations through the poloidal velocity, which splits into the poloidal projection of the parallel velocity and the perpendicular velocity

$$
v_{\theta}=\frac{B_{\theta}}{B} v_{\|}+v_{\perp} .
$$

For simplicity we take $B_{\phi} \approx B$. The drift which enters in the fluid equations is the $E \times B$ drift. The part of the perpendicular velocity due to the diamagnetic drift is largely divergence-free and is thus not connected to particle transport. The part of the diamagnetic drift which is not divergence-free is not taken into account in this model. The drift velocity can thus be estimated from

$$
v_{\perp}=-3.0 \frac{1}{H_{r}} \frac{k T_{\mathrm{e}}}{e B \lambda_{\mathrm{e}}}
$$

where we assume that the plasma potential in the SOL is determined by the sheath potential $\Phi \approx 3.0 k T_{\mathrm{e}}$ (see, e.g. [16]).

The boundary conditions at the two limiter sides $(\theta=$ $0,2 \pi)$ are for the poloidal velocity and the poloidal heat fluxes (given for example in [14])

$$
\begin{gathered}
v_{\theta}(0,2 \pi)= \pm \frac{B_{\theta}}{B} c_{\mathrm{s}}, \\
Q_{\theta \mathrm{e}}(0,2 \pi)= \pm \frac{B_{\theta}}{B} c_{\mathrm{s}} \gamma_{\mathrm{e}} n k T_{\mathrm{e}}, \\
Q_{\theta \mathrm{i}}(0,2 \pi)= \pm \frac{B_{\theta}}{B} c_{\mathrm{s}} \gamma_{\mathrm{i}} n k T_{\mathrm{i}},
\end{gathered}
$$

with $\gamma_{\mathrm{i}}=3.5$ and $\gamma_{\mathrm{e}}=5.0$.

Beside the usual parameters like magnetic field, beta poloidal and so on, there are three important control parameters which have to be prescribed. The density is controlled by the poloidal averaged density at the LCFS. This poloidal average might vary like for example during the plasma current scan. The e-folding length of the temperatures, which is taken to be the same for electrons and ions $\left(\lambda_{\mathrm{i}}=\lambda_{\mathrm{e}}\right)$, is needed to calculate the drift velocity. We keep it fixed at $\lambda_{\mathrm{e}}=50 \mathrm{~mm}$ for all $q$, which is in agreement with the experimental result. The temperatures are controlled by the radial heat flux to the SOL:

$$
P_{\mathrm{SOL}}=2 \pi a R_{0} \int_{0}^{2 \pi} \frac{1}{H_{r}}\left(Q_{\mathrm{e}}+Q_{\mathrm{i}}\right) \mathrm{d} \theta,
$$

which goes half to the electrons and half to the ions. The total heating power in the discussed discharges is $P_{\text {tot }}=1580 \mathrm{~kW}$. The radiated power is about $P_{\text {rad }}=800 \mathrm{~kW}$. Thus we have taken the fraction of the heating power which goes to the SOL to be $P_{\mathrm{SOL}}=780 \mathrm{~kW}$.

\subsection{Neutrals}

The neutrals are modelled in a very simplified way. However, the aim of the model is not to analyse in detail the neutral transport, but to get some idea of how drifts and particle sources influence each other. The main source for neutral particles in the edge of TEXTOR is the toroidal belt limiter. We distinguish between two types of neutrals, slow atoms with a temperature of $T_{0 \mathrm{~s}}=5 \mathrm{eV}$ and a fraction $f_{\mathrm{cx}}$ of fast atoms produced by charge exchange with $T_{0 \mathrm{f}}=T_{\mathrm{i}}$. Thus the neutral density is split into charge exchange neutrals and thermal neutrals: $n_{0}=f_{\mathrm{cx}} n_{0 \mathrm{f}}+\left(1-f_{\mathrm{cx}}\right) n_{0 \mathrm{~s}}$. For simplicity, the density of both species decays in poloidal direction from the limiter exponentially with the ionization lengths $\lambda_{0 \mathrm{~s}}$ and $\lambda_{0 \mathrm{f}}$ defined by

$$
\begin{gathered}
\int_{0}^{\lambda_{0 \mathrm{~s}}} n a \mathrm{~d} \theta=\left(\frac{k T_{0 \mathrm{~s}}}{m\left\langle\sigma_{\mathrm{i}} v\right\rangle^{2}}\right)^{0.5}, \\
\int_{0}^{\lambda_{0 \mathrm{f}}} n a \mathrm{~d} \theta=\left(\frac{k T_{\mathrm{i}}}{m\left\langle\sigma_{\mathrm{i}} v\right\rangle\left\langle\sigma_{\mathrm{cx}} v\right\rangle}\right)^{0.5} .
\end{gathered}
$$

The rate coefficients for ionization $\left\langle\sigma_{\mathrm{i}} v\right\rangle$ and for charge exchange $\left\langle\sigma_{\mathrm{cx}} v\right\rangle$ are chosen to be $3 \times 10^{-14} \mathrm{~m}^{3} \mathrm{~s}^{-1}$. They do not vary significantly in the temperature range we discuss here. The recycling coefficient $R_{\mathrm{SOL}}$, which gives the fraction of neutrals ionized in the SOL, is defined by

$$
\int_{0}^{2 \pi} S_{\mathrm{p}} a \mathrm{~d} \theta=R_{\mathrm{SOL}}\left(\Gamma_{\theta}(2 \pi)-\Gamma_{\theta}(0)\right),
$$

where $\Gamma_{\theta}=n v_{\theta}$. The particle source term due to ionization is $S_{\mathrm{p}}=n n_{0}\left\langle\sigma_{\mathrm{i}} v\right\rangle$, the momentum source reads like $S_{\mathrm{m}}=$ $-v_{\|} n n_{0}\left\langle\sigma_{\mathrm{cx}} v\right\rangle$. The directed velocity of the slow neutrals can be neglected in $S_{\mathrm{m}}$, the velocity of the charge exchange neutrals is not directed and does therefore not contribute. We take for all calculations a recycling coefficient of $R_{\mathrm{SOL}}=0.75$ and a fraction of charge exchange neutrals of $f_{\mathrm{cx}}=0.25$.

\subsection{Poloidal profiles}

An example of the poloidal profiles of the plasma parameters at the LCFS from our model is given in figure 6. Shown is the case for $I_{\mathrm{P}}=350 \mathrm{kA}$ and $B_{\phi}=2.25 \mathrm{~T}$, which are the standard values for TEXTOR. We compare the curves for normal field configuration (solid lines) with the results for the reversed configuration (dashed lines). The poloidal drift velocity is in the range of $v_{\perp}= \pm(600-1800) \mathrm{m} \mathrm{s}^{-1}$. Due to the Shafranov shift and the poloidal variation of the toroidal magnetic field, $v_{\perp}$ is higher at the LFS and lower at the HFS.

From the high parallel heat conductivity of the electrons, it is clear that $T_{\mathrm{e}}$ does not vary much with poloidal angle $\theta$. In contrast, the ion temperature varies by about $30 \mathrm{eV}$. Both temperatures show only a very slight change with field reversal. The density profile tends to peak in the vicinity of the limiter where the particle sources are located. This peaking is more pronounced at the limiter edge with its face towards the bottom of the machine. This asymmetry is caused by the Shafranov shift. Both, particle and energy input into the SOL, are higher at the LFS $(\theta=0.25 \pi)$. But since a significant fraction of the neutrals are ionized near the limiter in the SOL the particle input from inside the LCFS is less strong. The asymmetric energy input dominates and the peak of the temperature profile is shifted towards the LFS. Due to the pressure balance, the density tends to be higher near the bottom of the machine $(\theta=1.75 \pi)$. The field reversal gives rise to a change in the density in the vicinity of the limiter up to $20 \%$. It tends to increase the density at the bottom and decreases it at the LFS.

The strong change in the parallel velocity is determined by the boundary condition at the limiter. In contrast, the poloidal velocity changes only slightly. Thus the particle transport is 
(a) 120

(b)

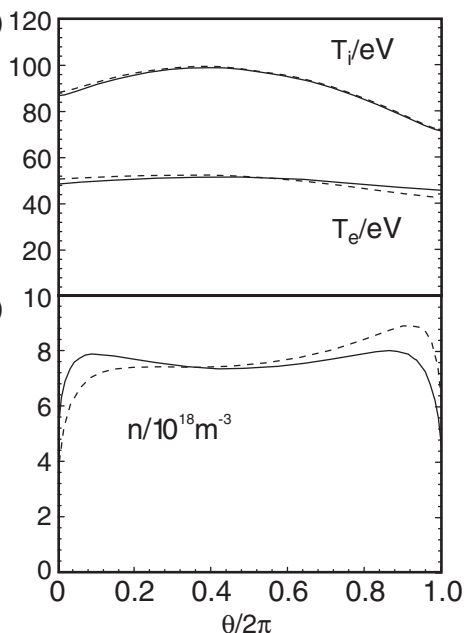

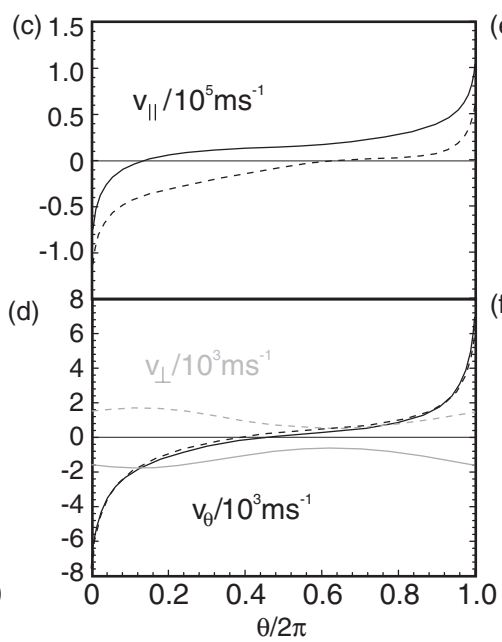

Figure 6. Solution of the model equations: poloidal profiles of $(a)$ the electron and ion temperature, $(b)$ the density, $(c)$ the parallel velocity, $(d)$ the perpendicular drift velocity and the poloidal velocity, $(e)$ the source due to ionization, and $(f)$ the perpendicular electric field in the SOL. Solid lines refer to the normal magnetic field configuration $\nabla B \downarrow$ and dashed lines refer to the reversed case $\nabla B \uparrow$.

not strongly affected by the field reversal due to the relative low drift velocity. The particle sources inside the SOL at the limiter tend to flatten the poloidal profile of $v_{\|}$further away from the limiter. We come back to this point in section 4.4 .

As we can see from figure $6(e)$, the particle sources change according to the changes in the particle flux in reversed configuration. These local particle sources enhance the density variation near the limiter with field reversal.

The perpendicular electric field is calculated from the electron momentum equation [13]:

$$
E_{\perp} \approx-\frac{k T_{\mathrm{e}}}{e n B_{\phi}} \frac{\partial n}{a \partial \theta}-\frac{1.71}{e B_{\phi}} \frac{\partial\left(k T_{\mathrm{e}}\right)}{a \partial \theta} .
$$

As we did in the whole model, we also neglect any currents which might flow in the SOL in this estimate. The role of the electric field concerning radial drift fluxes is discussed in section 4.2 .

\section{Discussion}

So far, we reported on the experimental results and introduced our SOL model. We will now compare the model predictions with the experiment. The model can give us information about the plasma properties at the LCFS and the effects on the radial structure, i.e. the SOL width, are discussed on the basis of analytical estimates.

\subsection{Density and temperature}

We go back to figure 2 and compare the measured $n, T_{\mathrm{e}}$ and $T_{\mathrm{i}}$ with the model values. In the model, the poloidal average of the SOL density is specified such as to fit the measured density at the LFS. The temperatures are determined by the power input $P_{\mathrm{SOL}}$ and the sheath heat transmission factors. Cooling by neutrals is less dominant and nearly all the power reaches the limiter. Both, $T_{\mathrm{e}}$ and $T_{\mathrm{i}}$, fit well with the measured values. The reader should keep in mind that $T_{\mathrm{i}}$ was measured $6 \mathrm{~mm}$ inside the SOL, whereas the other quantities-measured and modelled-are given at the LCFS. Taking this into account,
$T_{\mathrm{i}}$ at the LCFS is $13 \%$ higher than the value given in figure 2 $\left(\lambda_{\mathrm{i}} \approx 50 \mathrm{~mm}\right)$.

In the case of the plasma current scan, the modelling result for the increase of the density is quite artificial: we simply increased the specified average density. This density increase is - as pointed out earlier - due to changes of the core density profile. Such effects can of course not be modelled with our SOL model. The modelled temperatures decrease with lower plasma current due to the density variation. Since we keep the power input constant, we expect to find a $T \sim n^{-2 / 3}$ dependence. This dependence was found experimentally for density ramps at TEXTOR (see, e.g. [10]). For the current scan, we find no such tendency of the measured temperature. However, the position of the plasma column changes during the $I_{\mathrm{P}}$ scan. One might speculate that this change in the geometry might have influence on the edge parameter. The modelled temperature for the $B_{\phi}$ scan shows a slight increase. This can be attributed to the fact that the effective collection area of the limiter $A_{\text {eff }}=2 \pi a \lambda_{q} / q$ decreases with increasing $q$. Thus, the temperature has to increase in order to keep the power transported to the limiter constant.

As stated earlier, the field reversal has a stronger effect on the density than on the temperatures. This is found in the model as well as in the experiment. The decrease of the density at the LFS is well seen in the model results and is roughly of the same order as in the experiment.

\subsection{SOL width}

The SOL width is extracted from the balance between the radial particle flux into the SOL, the particle sources in the SOL and the flux carried to the limiter

$\int_{0}^{\infty} \Gamma_{\theta}(2 \pi)-\Gamma_{\theta}(0) \mathrm{d} r=a \int_{0}^{2 \pi} \Gamma_{r} \mathrm{~d} \theta+a \int_{0}^{2 \pi} \int_{0}^{\infty} S_{\mathrm{p}} \mathrm{d} r \mathrm{~d} \theta$.

Neglecting any particle sources and drift motions one finds with $\Gamma_{\theta}(2 \pi)=-\Gamma_{\theta}(0)=0.5 n c_{\mathrm{s}} B_{\theta} / B$ the well-known 
formula for the density decay length

$$
\lambda_{n}^{(0)}=\sqrt{\frac{D L_{\mathrm{c}}}{c_{\mathrm{s}}}}
$$

which is a function of the radial diffusion coefficient $D$, the connection length $L_{\mathrm{c}}=2 \pi R q=2 \pi a B / B_{\theta}$ and the sound speed $c_{\mathrm{s}}$. This 'first order' estimate might be modified by several quantities which we will discuss in the following.

4.2.1. Poloidal drifts. The derivation of equation (26) needs an assumption about the poloidal flux at the limiter, especially about the density at the limiter. In the above derivation, we used $n(\mathrm{lim}) / n=0.5$. Poloidal drifts change the poloidal flux to the limiter and lead to asymmetric values of the density at each side of the limiter. We can estimate the fluxes at the limiter by solving the particle conservation and momentum equation (equations (1) and (2)) for the isothermal case without sources. Metric coefficients and the poloidal variation of $B$ are neglected. In that way it is possible to derive expressions for the density and the velocity:

$$
\begin{gathered}
\frac{\partial n}{a \partial \theta}=\frac{B}{B_{\theta}} \frac{\partial \Gamma_{r}}{\partial r} \frac{2 v_{\|}+v_{\perp} B / B_{\theta}}{c_{\mathrm{s}}^{2}-\left(v_{\|}+v_{\perp} B / B_{\theta}\right)^{2}}, \\
\frac{\partial v_{\|}}{a \partial \theta}=\frac{B}{n B_{\theta}} \frac{\partial \Gamma_{r}}{\partial r} \frac{c_{\mathrm{s}}^{2}+v_{\|}\left(v_{\|}+v_{\perp} B / B_{\theta}\right)}{\left(v_{\|}+v_{\perp} B / B_{\theta}\right)^{2}-c_{\mathrm{s}}^{2}} .
\end{gathered}
$$

These equations have been discussed already in [17], they have been used for the deduction of poloidal Mach numbers from probe measurements [18] and they have been used in [19] for the interpretation of biasing experiments. The radial derivative of the radial flux is taken to be proportional to the density $n$ (see equation (6)). From the equations (27) and (28) an analytical expression for the density as a function of the parallel velocity can be given:

$$
\frac{n\left(v_{\|}\right)}{n}=\frac{c_{\mathrm{s}}^{2}}{c_{\mathrm{s}}^{2}+v_{\|}\left(v_{\|}+v_{\perp} B / B_{\theta}\right)} .
$$

With the boundary condition at the limiter for the parallel velocity $v_{\|}(\mathrm{lim})= \pm c_{\mathrm{s}}-v_{\perp} B / B_{\theta}$ we get (cf equation (18.40) of [16])

$$
\frac{n(\lim )}{n}=\frac{1}{2 \pm v_{\perp} B / c_{\mathrm{s}} B_{\theta}} .
$$

Inserting these densities into the flux balance (25) we get for the SOL width

$$
\frac{\lambda_{n}}{\lambda_{n}^{(0)}}=\left(\frac{1}{2+v_{\perp} B / B_{\theta} c_{\mathrm{s}}}+\frac{1}{2-v_{\perp} B / B_{\theta} c_{\mathrm{s}}}\right)^{-0.5} .
$$

The poloidal drift reduces the SOL width independently of the drift direction. The poloidal drift velocities are of the order $v_{\perp}= \pm 2 \times 10^{3} \mathrm{~m} \mathrm{~s}^{-1}$ and the magnetic field ratio $B_{\theta} / B$ is typically around 0.08 . This will reduce the SOL width according to (31) by only $1 \%$.

The radial dependence of the poloidal drift gives rise to a change in the e-folding length, which is not poloidally uniform. We have seen that the field reversal gives rise to a density change $\Delta n$ at a certain poloidal position. The density at this position without any drift effects is increased or decreased if the drift is 'switched on' $n \rightarrow n+\Delta n / 2$. The e-folding length of the density changes according to this like

$$
\frac{1}{\lambda_{n}}=\frac{1}{n} \frac{\partial n}{\partial r} \rightarrow \frac{1}{n} \frac{\partial(n+\Delta n / 2)}{\partial r}=\frac{1}{\lambda_{n}}+\frac{1}{2 n} \frac{\partial \Delta n}{\partial r} .
$$

If we assume that the $\Delta n$ is proportional to the drift velocity $v_{\perp}$ and thus proportional to the electron temperature, we get for the change in $\lambda_{n}$ :

$$
\frac{\Delta \lambda_{n}}{\lambda_{n}} \approx-\frac{\Delta n}{n} \frac{\lambda_{n}}{\lambda_{\mathrm{e}}}
$$

With $\Delta n / n$ of about $10 \%$ and $\lambda_{\mathrm{e}}=50 \mathrm{~mm}$, this results in a change in $\lambda_{n}$ at the LFS of about $2-3 \%$. We expect from these considerations an increase of the SOL width at the LFS in reversed configuration, which is in agreement with the experimental result.

4.2.2. Radial drift. Poloidal electric fields lead to radial $E \times B$ drifts. The radial fluxes connected to these drifts can become of the same order as the fluxes driven by the anomalous diffusive transport. As we can see from figure $6(f)$, the poloidal electric field increases strongly in the vicinity of the limiter and we expect there a non-negligible influence of these radial drifts.

The radial particle flux connected to this drift is

$$
\Gamma_{r}^{E}=n \frac{E_{\perp}}{B} .
$$

The diffusive flux is given by equation (5) (we keep here the metric coefficient constant), so that the ratio of these fluxes is

$$
\frac{\Gamma_{r}^{E}}{\Gamma_{r}^{D}}=\frac{\lambda_{n} E_{\perp}}{D B} .
$$

If we fill in typical values like $\lambda_{n}=15 \mathrm{~mm}, B=2 \mathrm{~T}$ and $D=0.2 \mathrm{~m}^{2} \mathrm{~s}^{-1}$, this ratio becomes unity if $E_{\perp}$ exceeds about $25 \mathrm{~V} \mathrm{~m}^{-1}$. Although we might expect that parallel currents will attenuate the electric field, we see that the radial fluxes can play a non-negligible role in the vicinity of the limiter. Moreover, these radial drifts might enhance the asymmetry in the SOL, since they tend to decrease the radial transport at one limiter side, whereas they increase the radial transport at the other limiter side. However, any further analysis concerning these radial drifts and their influence on the radial structure needs to solve the full two-dimensional set of equations.

4.2.3. Ionization. We distinguish between two types of ionization sources: a homogeneous distribution of neutrals in the SOL and a localized ionization source near the limiter. For the first type we introduce the source term $S_{\mathrm{p}}=n n_{0}\left\langle\sigma_{\mathrm{i}} v\right\rangle$ with constant $n_{0}$ into equation (25) which gives us:

$$
\frac{\lambda_{n}}{\lambda_{n}^{(0)}}=\left(1-\frac{L_{\mathrm{c}} n_{0}\left\langle\sigma_{\mathrm{i}} v\right\rangle}{c_{\mathrm{s}}}\right)^{-0.5} .
$$

A homogenous neutral background might result from neutrals starting at nearby wall structure. Measurements with laserinduced fluorescence in the TEXTOR SOL have shown that a radially constant distribution of neutrals exists with 
$n_{0} \leqslant 5 \times 10^{15} \mathrm{~m}^{-3}$ [20]. Using the above equation with typical values for the discussed discharges-a connection length of $L_{\mathrm{c}}=40 \mathrm{~m}$ and sound speed of $c_{\mathrm{s}}=10^{5} \mathrm{~m} \mathrm{~s}^{-1}$ we find that the SOL width is changed by less than $3 \%$ by these neutrals.

The dominant source for neutral particles in TEXTOR is the toroidal belt limiter. For this localized neutral distribution we assume an exponential decay in poloidal direction with a penetration depth $\lambda_{0}$ defined by the neutral velocity $v_{0}$ (see section 3). Exploiting again equation (25) together with the definition of the recycling coefficient $R_{\mathrm{SOL}}$ (23) we get (cf [21])

$$
\lambda_{n}^{2}=\frac{L_{\mathrm{c}} D}{c_{\mathrm{s}}\left(1-R_{\mathrm{SOL}}\right)}
$$

and the SOL width ratio is

$$
\frac{\lambda_{n}}{\lambda_{n}^{(0)}}=\left(1-R_{\mathrm{SOL}}\right)^{-0.5} \text {. }
$$

The neutrals are in both cases an additional particle source to the radial particle influx. The SOL plasma reacts by an increase of the SOL width to reduce the radial influx and to increase the total parallel flux to the limiter. The localized source influences additionally the parallel velocity $v_{\|}$. The poloidal gradient of the parallel velocity is reduced outside the source region and steep poloidal gradients of $v_{\|}$are found inside the source region (cf figure 6).

\subsection{Transport coefficients}

The diffusion coefficient can be extracted by fitting the SOL width derived from the SOL model to the experimental values as good as possible (cf figure 3 ). The modelled $\lambda_{n}$ does strongly depend on the recycling coefficient $R_{\mathrm{SOL}}$ according to formula (38). With the chosen value of $R_{\mathrm{SOL}}=0.75 \mathrm{a}$ diffusion coefficient of $D=0.2 \mathrm{~m}^{2} \mathrm{~s}^{-1}$ gives an acceptable agreement with the measured $\lambda_{n}$. With this constant diffusion coefficient the increase of $\lambda_{n}$ with the safety factor is achieved in the model by the increase of the connection length alone. Without neutral ionization inside the SOL, we would get a diffusion coefficient four times larger compared to this value. The ratio between the diffusion coefficient derived without ionization $\left(D^{(0)}\right)$ and the diffusion coefficient with ionization (D) scales with $R_{\mathrm{SOL}}$ like $D^{(0)} / D=1-R_{\mathrm{SOL}}$.

A diffusion coefficient based on the simple formula (26) would give a dependence of $D$ on the magnetic field orientation. In the considerations above, we tried to analyse possible mechanisms, which have influence on $\lambda_{n}$. During reversed $B$, many of these mechanisms might change their influence which could lead to the increase in $\lambda_{n}$. However, so far we are not able to choose one of these mechanisms to be the dominant one.

The ratio between the radial electron heat and particle diffusion coefficient $\chi_{\mathrm{e}} / D$ can be extracted from the balance of the heat fluxes

$$
\int_{0}^{\infty} Q_{\theta}(2 \pi)-Q_{\theta}(0) \mathrm{d} r=a \int_{0}^{2 \pi} Q_{r} \mathrm{~d} \theta .
$$

Any heat sinks or sources in the SOL are neglected here. We insert the poloidal electron heat flux at the limiter as given in

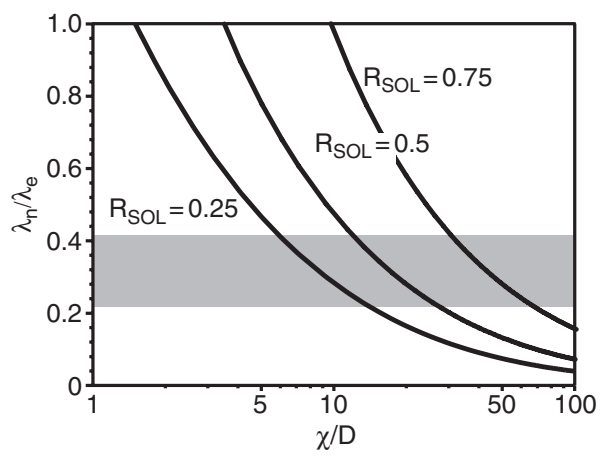

Figure 7. Ratio of the e-folding lengths versus the ratio of the transport coefficients for different recycling coefficients $R_{\mathrm{SOL}}$. The measured values are indicated by the grey box.

equation (18) and the radial heat flux given with equation (7) (metric coefficients are kept constant). With $\kappa_{r}=n \chi_{\mathrm{e}}$ we get together with equation (38) (cf [22])

$$
\frac{\gamma_{\mathrm{e}}}{1-R_{\mathrm{SOL}}}=\left(1+\frac{\lambda_{n}}{\lambda_{\mathrm{e}}}\right)\left(\frac{5}{2}+\frac{\chi_{\mathrm{e}}}{D} \frac{\lambda_{n}}{\lambda_{\mathrm{e}}}\right) .
$$

From figure 7 it is obvious that a precise knowledge of $R_{\mathrm{SOL}}$ is necessary for the determination of $\chi_{\mathrm{e}} / D$. The electron temperature e-folding length $\lambda_{\mathrm{e}}$ (figure 3 ) in the SOL of TEXTOR is about a factor of 3 larger than $\lambda_{n}$. The measured ratio $\lambda_{n} / \lambda_{\mathrm{e}}$ is indicated in figure 7 . The measurement error and especially the uncertainty in $R_{\text {SOL }}$ gives a broad band of values for $\chi_{\mathrm{e}} / D$ between 5 and 80 . We do not discuss the transport coefficient for the ions, since we have no measurement of $\lambda_{i}$, but the same statement is valid for $\chi_{\mathrm{i}} / D$.

\subsection{Poloidal rotation}

The SOL model is not able to describe impurity transport in a hydrogen plasma. Nevertheless a comparison between the modelled velocities and the measured $\mathrm{C}^{6+}$ velocities can be done if we keep in mind the following restrictions. The diamagnetic drift due to the pressure gradient depends on the ion charge, leading to a smaller poloidal drift for $\mathrm{C}^{6+}$. A steeper $\mathrm{C}^{6+}$ density gradient-as measured by CXRS with a fast Lithium beam [23] - might balance this reduction of the drift velocity to some extend. The parallel flow velocity of the carbon minority depends on the friction forces in the SOL. In the simple picture (without drift motions and sources) it is shown that the parallel flow velocity of carbon is-depending on the plasma parameter-between $15 \%$ and $40 \%$ slower with respect to the background plasma [24].

In the experiment we do not only measure the $E \times B$ drift plus the poloidal projection of the parallel velocity (what we called so far $v_{\theta}$ in the model), but additionally the diamagnetic drift velocity (the fact that the latter is (largely) divergence-free does not matter). Consequently the poloidal velocity deduced from the model as given in figure 4 is

$$
\hat{v}_{\theta}=v_{\theta}+v_{\perp}^{\mathrm{dia}} .
$$

The diamagnetic drift velocity is

$$
v_{\perp}^{\mathrm{dia}}=-\frac{1}{e n B} \frac{\partial p_{\mathrm{i}}}{\partial r}=\frac{k T_{\mathrm{i}}}{e B}\left(\frac{1}{\lambda_{n}}+\frac{1}{\lambda_{\mathrm{i}}}\right),
$$

and we take again $\lambda_{\mathrm{i}}=\lambda_{\mathrm{e}} \approx 50 \mathrm{~mm}$. 
As we see from figure $6(d)$, the poloidal velocity including only the $E \times B$ drift has nearly no dependence on the magnetic field orientation. In particular the direction of $v_{\theta}$ does not change in reversed $B$. It is the diamagnetic drift which leads to the strong change in $\hat{v}_{\theta}$ with reversal and the $v_{\theta}$ gives just rise to an offset on this total poloidal velocity.

\subsection{Poloidal asymmetries}

The strong variation of the density ratio $n$ (HFS) $/ n$ (LFS) with field reversal cannot be found with the modelled densities (figure 5). However, the tendency of an increased ratio in reversed $B$ configuration is reproduced by the model. Thus the influence of the poloidal $E \times B$ drift can be clearly seen, although this drift is too weak to explain the large poloidal asymmetry in the density. The density ratio between the two poloidal positions shows also that the local particle sources at the limiter are needed. They explain that the density tends to be larger near the limiter (LFS) compared to positions further away from the limiter (HFS).

\section{Summary}

The plasma parameters at the edge were measured with the thermal helium beam diagnostic. This diagnostic provides not only electron densities and temperatures, but can also be used for CXRS in the plasma edge for the determination of ion temperatures and velocities. We have analysed the dependence of these parameters in TEXTOR on the safety factor and magnetic field reversal.

The major effect of the safety factor is to control the connection length and the pitch angle of the field lines. The measurements show that these features are not the only driving forces on the edge parameters. During the current scan, the edge density shows a complete different behaviour with the safety factor compared to the magnetic field scan. This behaviour is attributed to changes in the core density profile: a different shape of the core profile affects the edge density since the line averaged density is kept constant. The density e-folding length is clearly a function of the connection length and increases with the safety factor. The temperature e-folding shows no explicit dependence on the safety factor.

Typical features connected with field reversal are the decrease of the edge density at the LFS, the increase of the density e-folding length at LFS and HFS and the strong difference in the density ratio between HFS and LFS. The measurement of the poloidal drift velocity of $\mathrm{C}^{6+}$ at the LFS gives evidence that drifts play a role in the plasma edge. The poloidal velocity changes sign with field reversal. However, the interpretation of this poloidal rotation is more complex since not only the poloidal drifts contribute, but also the poloidal projection of the parallel velocity.

In order to study the influence of the drifts on the plasma transport in the SOL in more detail, we introduced a simplified fluid model. The choice to use such a model was motivated by the endeavour to include drifts and neutrals into a fluid model, but to still keep it that simple that their main effects can easily be extracted.

The drift included in the transport equations is the poloidal $E \times B$ drift. With the model, we were able to reproduce the decrease of the edge density at the LFS with field reversal. The tendency to have higher densities in the vicinity of the limiter can be attributed to the local particle sources there. Although we compare the poloidal velocities of $\mathrm{D}^{+}$in the model with those of $\mathrm{C}^{6+}$ in the experiment, both show a change of sign with field reversal and the absolute values are of the same order. We see from the model that the $E \times B$ drift has a negligible effect on the convective poloidal velocity. Due to the contribution of the poloidal projection of the parallel velocity, the convective poloidal velocity does not change significantly with field reversal. Only if the diamagnetic drift is added to the poloidal velocity, the reversal of the poloidal rotation is achieved. A change in the density e-folding length with field reversal is not seen in the model results, since we keep the particle sources constant. However, the SOL width is affected by various mechanisms: for example the radial variation of the poloidal rotation, the influence of radial drifts on the radial particle transport, or a variation in the particle source distribution. Since all these effects make small contribution to the whole, the correct modelling and identification of these mechanisms is difficult.

Although, we still do not have a complete picture of how the density e-folding length is affected, it is clear that the experimentally observed change of $10-20 \%$ with field reversal has to be understood in order to get more accurate results for the transport coefficients. Some care is needed in calculating for example the diffusion coefficient, which varies about $20-45 \%$ with field reversal if calculated by the simple formula with no other effect taken into account. The calculation of the heat transport coefficients from e-folding lengths is also difficult, because these coefficients show in the simple estimates a very strong dependence on the fraction of neutrals that are ionized inside the SOL.

We can conclude from the comparison between model and experiment, that the poloidal $E \times B$ drift contributes to poloidal asymmetries in the SOL. These modelled asymmetries showcompared to the experiment- the right trend. But this drift only is not sufficient to explain the strong impact of the field reversal on the poloidal density distribution.

Further investigations on the velocity measurements have to be done in the future, since they give insight into the plasma rotation due to particle drifts. Possible differences between the flow velocities of background plasma and impurities should be taken into account. The simultaneous measurement of the poloidal and toroidal velocity components for a complete description of the SOL flows and drifts in TEXTOR would be a task of high interest.

\section{Acknowledgments}

The author would like to thank H. Gerhauser and R. Zagórski for many fruitful discussions and especially D. Reiser for his very helpful comments on modelling questions.

\section{References}

[1] Chankin A.V. et al 1994 Plasma Phys. Control. Fusion 36 1853

[2] Bonnin X. and Rowan W.L. 1999 Nucl. Fusion 391009

[3] Kaufmann M. et al 1993 Plasma Phys. Control. Fusion 35 B205 
[4] Samm U. et al 1991 Nucl. Fusion 311386

[5] Lehnen M., Brix M., Gerhauser H., Schweer B. and Zagorski R. 2001 J. Nucl. Mater. 290-293 663

[6] Erents S.K. et al 2000 Plasma Phys. Control. Fusion 42905

[7] Loarer T. et al 2001 Czech. J. Phys. 511021

[8] Porter G.D. et al 2003 Simulation of the effect of plasma flows in DIII-D, JET, and JT-60U J. Nucl. Mater to be published

[9] Chankin A.V. and Stangeby P.C. 2001 Nucl. Fusion 41421

[10] Schweer B., Brix M. and Lehnen M. 1999 J. Nucl. Mater. 266-269 673

[11] Bogen P. et al 1995 J. Nucl. Mater. 220-222 472

[12] Hey J.D., Lie Y.T., Rusbüldt D. and Hintz E. 1993 20th Eur Conf. Control. Fusion Plasma Phys. p 1111

[13] Braginskii S.I. 1965 Rev. Plasma Phys. 1205

[14] Baelmans M. 1994 Jül-Report Forschungszentrum Jülich Jül-2891, ISSN 0944-2952
[15] Soltwisch H. 1990 Jül-Report Forschungszentrum Jülich Jül-2339, ISSN 0366-0885

[16] Stangeby P.C. 2000 The Plasma Boundary of Magnetic Fusion Devices (Bristol: Institute of Physics Publishing) ISBN 0750305592

[17] Stangeby P.C. and Chankin A.V. 1996 Nucl. Fusion 36839

[18] van Goubergen H. et al 1999 Plasma Phys. Control. Fusion $41 \mathrm{~L} 17$

[19] Loarer T. et al 2000 Czech. J. Phys. 501441

[20] Mertens Ph. and Silz M. 1997 J. Nucl. Mater. 241-243 842

[21] Stangeby P.C. 1984 J. Nucl. Mater. 12155

[22] Stangeby P.C. and McCracken G.M. 1990 Nucl. Fusion 30 1225

[23] Schorn R.P. et al 1991 Appl. Phys. B 5271

[24] Tokar M.Z. 1994 Control. Plasma Phys. 34344 